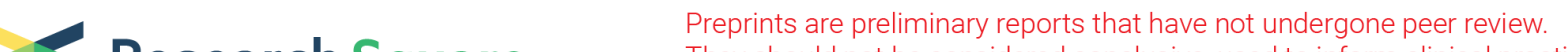 Research Square They should not be considered conclusive, used to inform clinical practice, or referenced by the media as validated information.
}

\section{A Clinical Study of Internet Gaming Disorder in Adolescents with Psychiatric Disorders}

\section{Praveen Sachan}

King George's Medical University

\section{Amit Arya}

King George Medical University: King George's Medical University

\section{Shweta Singh}

King George Medical University: King George's Medical University

\section{Pawan Kumar Gupta ( $\sim$ gpawan2008@gmail.com )}

King George Medical University: King George's Medical University https://orcid.org/0000-0001-75235988

\section{Vivek Agarwal}

King George Medical University: King George's Medical University

\section{Regular Article}

Keywords: Internet Gaming Disorders, IGDS, Phenomenology, Comorbidities

Posted Date: February 11th, 2021

DOI: https://doi.org/10.21203/rs.3.rs-175108/v1

License: (c) (i) This work is licensed under a Creative Commons Attribution 4.0 International License. Read Full License 


\section{Abstract}

Purpose-The severity of different IGD symptoms criteria has not been explored much. The study describes the phenomenology of IGD in Adolescents with comorbid psychiatric disorders attending child and adolescent psychiatry OPD.

Methods- Adolescents aged 13-16 years, who have attended child and adolescent Psychiatry OPD, of a tertiary care teaching hospital for psychiatric disorders, with a history of gaming and fulfilled criteria as per DSM-5 for IGD have been included. All the subjects have then assessed using semi-structured proforma, IGDS and K-SADS-PL.

Results- In the study Sample of 46 Adolescents most common IGDS criteria was "Conflict" and least common criterion was "Tolerance". IGDS mean Score of "Conflict" was the highest while it was the least for "Displacement". "Escape "and "Deception" were significantly higher for females. "Displacement" significantly higher for Urban domicile and Online mode of gaming. "Persistence" was significantly higher for those who were gaming on personal devices and playing MMORPGs. Psychiatric disorders associated with IGD were ODD (46.66\%), Dissociative disorder (24.44\%), ADHD (17.77\%) and Depressive disorder (11.11\%). Phenomenology of IGD was comparable across all psychiatric comorbidities except subjects with dissociative disorders, who had significantly higher scores for "Escape" than ADHD and Depression. Also, subjects with ODD had significantly higher scores for "Displacement" than the subjects with Dissociative disorder in terms of IGDS scores.

Conclusion: Significant differences in the severity of DSM-5-IGD symptoms criteria is found in terms of gender, domicile, gaming genre (MMORPGs), accessibility of smartphones, online/offline modes of gaming and the associated psychiatric comorbidity.

\subsection{Introduction}

Video games are a popular entertainment method among all age groups, especially in adolescents and young adults, which has successfully captured the interests of many over the years since its launch. Gaming Disorder is considered a behavioural addiction (BA)(M. D. Griffiths et al., 2013; Leeman \& Potenza, 2013). Multiple similarities have been described between addictive gaming and other addictions such as substance dependence. Substance-related and BA affect the 'reward circuit' (Dong \& Potenza, 2014; Weinstein \& Lejoyeux, 2015). Many online addictions have been identified to date; however, gaming addiction has become a topic of continually evolving research because it can affect both physical and mental health.

The fifth edition of Diagnostic and Statistical Manual (DSM-5) defines Internet Gaming Disorder (IGD) as the "Persistent and recurrent use of the Internet to engage in games, often with other players, leading to clinically significant impairment or distress," as indicated by five (or more) out of nine proposed items following in 12 months. The APA has identified IGD as a new potential psychiatric condition and acknowledged that little is understood about the prevalence, validity of diagnostic criteria, and 
significance for Internet gaming condition. Currently, the prevalence of IGD in adults $0.5-27.5 \%$ (Mihara \& Higuchi, 2017). Prevalence rates of IGD vary widely with studies from different countries across the world. Variations may be due to differences in assessment tools, study population (Daria Joanna Kuss \& Griffiths, 2012) and diagnostic criteria of IGD. Prevalence rates are highest in Eastern Asian countries, and male adolescents aged 12 years to 20 years (Association, 2013). Male adolescents have a higher chance of being predisposed to gaming disorder. It is not clear that the gamer's age influences gaming behaviours, but adolescents are found to have the strongest influence of IGD (M. Griffiths \& Wood, 2000). Concerning IGD, low parental support (F. Rehbein et al., 2015), elevated use of video games by parents, divorce, or separation of parents(Batthyany et al., 2009), and single-parent families (Florian Rehbein \& Baier, 2013) have been associated with IGD. Many other correlates have also been identified as predictors of IGD such as personality especially impulsivity(Jiang \& Leung, 2012) conduct problems, low level of outdoor sports involvement, limited social interaction, and low self-esteem. Internet gaming can be comorbid with anxiety disorders(D. A. Gentile et al., 2011), depression(D. A. Gentile et al., 2011), behavioural disorders, social phobia, autism spectrum disorder(Mazurek \& Engelhardt, 2013), attentiondeficit hyperactivity disorder (ADHD)(Bioulac et al., 2008; D. Gentile, 2009; Walther et al., 2012), obsessive-compulsive disorder, and personality disorders(Andreassen et al., 2016; Chan \& Rabinowitz, 2006; Ferguson et al., 2011; Ko et al., 2006).

Differing opinions are also present in terms of symptom criteria of internet gaming, and the primary limitation is the lack of homogeneity in research methods. There are controversies about the validity of IGD criteria. Studies investigated the validity of the DSM-5 criteria. Some of them were more suited than others: 'escape', and 'preoccupation' were judged as less suitable(Király et al., 2015; Ko et al., 2014; Florian Rehbein et al., 2015). 'Deception' in the study of Ko et al. (Ko et al., 2014) was less suited for identifying IGD, whereas other studies (Pontes \& Griffiths, 2015; Florian Rehbein et al., 2015) found the opposite. 'Giving up other activities' and 'tolerance' [22,24], as well as 'withdrawal' (Florian Rehbein et al., 2015) and 'loss of control' (Király et al., 2015), were also debated. Also, Ko et al. (Ko et al., 2014) suggested 'craving' as a candidate criterion. Another controversy is the problem of a cut-off level of symptoms because of the lack of standard defining criteria for IGD(Király et al., 2015; Ko et al., 2014; Florian Rehbein et al., 2015). Despite the increasing number of studies in IGD, the phenomenology lacks a consensual definition. The APA included the IGD under section III of the DSM-5, i.e., conditions for further research(Association, 2013), not intended for clinical use, but to provide uniform guidelines for future studies. These criteria required further investigation to be used for clinical purposes.

Given the lack of clinical studies concerning IGD, exploring clinical samples' characteristics with IGD is much required. Adolescent clinical samples will help delineate the gaming disorder construct and help in future treatment plans. Indian studies are needed. Hence, this study was planned to see the phenomenology of Internet gaming in adolescents presenting to Child and adolescent psychiatry OPD of a tertiary care institute of north India.

\subsection{Material And Methods}


Adolescents (13-16 years) presenting to the child and adolescent psychiatry OPD, KGMU, Lucknow, UP, primarily for psychiatric disorders along with chief complaints of video gaming (Online OR Offline) in initial clinical interview were screened for selection criteria. A details clinical interviewing was conducted using IGDS-27 with the adolescents and the available parent and the diagnosis of IGD was made by consensus of the investigator (PS) and either of a consultant psychiatrist (PKG, VA, AA) involved in the study. Informed consent of parent and ascent by the adolescents have been taken. Inclusion criteria were availability of at least one of a parent as a reliable informant. Exclusion criteria were Intellectual disability, patients with suicidal risk, and the presence of medical comorbidity that require urgent medical attention, to avoid risking patients' health or unreliable measurements. A sample size of 45 was calculated by using the standard formula. Total 74 subjects have been screened out of which 46 subjects fulfilled the selection criteria and 28 subject were excluded. The reason for non-exclusion was not fulfilling IGDS criteria $(n=10)$, not fulfilling age criteria $(n=14)$, one was diagnosed Mental retardation and 3 subjects refused to give consent.

IQ was derived from percentile scores of intelligences as measured by a faculty of clinical psychology by using Raven's Standard Progressive Matrices (SPM) (Domino \& Domino, 2006) and Raven's Coloured Progressive Matrices (CPM) (Domino \& Domino, 2006). Kiddie Schedule for affective disorders and schizophrenia for school-age children-present and lifetime versions (K-SADS-PL)(Kaufman et al., 1997) was applied to assess psychiatric disorders. Conditions that are not included in K-SADS-PL were assessed by clinical evaluation. Information regarding identification data, demographic details, chief complaints, history of present illness, past illness, family history, personal history and physical examination, and information on the gaming and related variables was obtained on a semi-structured proforma. Internet Gaming Disorder Scale (IGDS 27 item)(Lemmens et al., 2015) was applied to assess the symptoms and the severity of IGD. Data obtained was analyzed using Unpaired $t$-test, ANOVA and Tukey's post hoc test.

\subsection{Results}

The study sample was a purposive sample consisting of 46 subjects (mean age $14.02 \pm 1.20$ years) who were included in the study from 11th October 2019 to 31st August 2020. (table.1)

Table 1: Socio-demographic profile of subjects $(\mathrm{N}=46)$ 


\begin{tabular}{|l|c|c|}
\hline \multicolumn{1}{|c|}{ Variables } & Category & $\begin{array}{c}\text { Subjects } \\
\text { N=46 (\%) }\end{array}$ \\
\hline \multirow{2}{*}{ Gender } & Male & $41(89.13)$ \\
\cline { 2 - 3 } & Female & $05(10.87)$ \\
\hline \multirow{2}{*}{ Fomicile } & Urban & $29(63.00)$ \\
\cline { 2 - 3 } & Rural & $17(37.00)$ \\
\hline \multirow{2}{*}{ IQ of study subjects } & Nuclear & $34(73.90)$ \\
\cline { 2 - 3 } (Mean \pm SD=94.82 \pm 7.20$)$ & Joint & $12(26.10)$ \\
\cline { 2 - 3 } & $80-89$ & $03(6.52)$ \\
\cline { 2 - 3 } & $90-109$ & $38(82.60)$ \\
\cline { 2 - 3 } & $>110$ & $05(10.86)$ \\
\hline
\end{tabular}

According to the present study, Smartphones (91.31\%) were the most common devices with the online mode of video gaming (65.21\%), belonging to the parents $(65.21 \%)$ and the adolescents themselves' (28.26\%). Massively Multiplayer Online Role-Playing Games (MMORPGs) were the commonest games that involved $58.69 \%$ of subjects.

Most common IGDS criteria was Conflict (95.65\%) followed by Preoccupation (80.43\%), Withdrawal (76.08\%), Persistence (69.56\%), Problem (67.39\%), Displacement (56.52\%), Deception (54.34\%), Escape $(47.82 \%)$ and the least common criteria was Tolerance $(41.30 \%)$, but in terms of severity measured by the mean IGDS scores, it was highest for Conflict $(9.83 \pm 2.33)$ followed by Persistence $(9.78 \pm 1.97)$, Withdrawal (8.25 \pm 1.78$)$, Tolerance $(7.88 \pm 1.58 \%)$, Preoccupation $(7.81 \pm 2.48 \%)$, problem $(7.77 \pm 1.78 \%)$, Deception (7.24 \pm 2.04$)$, Escape $(6.95 \pm 1.55 \%)$ and the least for Displacement $(6.88 \pm 1.55 \%)$. ODD $(46.66 \%)$ was the most common psychiatric disorder associated with IGD followed by Dissociative disorder (24.44\%), ADHD (17.77\%) and Depressive disorder (11.11\%).

Subjects diagnosed with Dissociative disorder have significantly higher scores for "Escape" criteria than subjects diagnosed with ADHD and Depression ( $p=0.005$ and $p=0.007$ respectively). Subjects diagnosed with ODD have significantly higher scores for "Displacement" criteria $(p=0.0494)$ than subjects diagnosed with Dissociative disorder. 
The IGDS scores of "Escape" ( $p=0.0318)$ and "Deception" ( $p=0.0318)$ criteria were significantly more in females than males. "Displacement" criterion was significantly higher for urban subjects $(p=0.0005)$ involved with online gaming $(p=0.0023)$. "Persistence" criterion was significantly higher for subjects playing games on personal devices $(p=0.031)$ and MMORPGs $(p=0.0342)$.

\subsection{Discussion}

The present study explored the phenomenology of DSM-5 Internet Gaming Disorders (IGD-DSM-5) and its associated psychiatric comorbidities among adolescents (13-16 Years) attending to the child and adolescent psychiatry OPD KGMU Lucknow UP for other psychiatric disorders.

In the present study, most common IGDS criteria was Conflict (95.65\%) followed by Preoccupation (80.43\%), Withdrawal (76.08\%), Persistence (69.56\%), Problem (67.39\%), Displacement (56.52\%), Deception (54.34\%), Escape (47.82\%) and the least common criteria was Tolerance $(41.30 \%)$. Their proportions of IGDS criteria found in the present study were different from the previous study of 824 adolescents conducted by king et al(King \& Delfabbro, 2016). which have reported Escape (96\%), Preoccupation (92\%), Tolerance (77\%), and continued use despite harm $(77 \%)$ as the common criteria. The difference in the finding of this present study may be due to the sampling effect and the comorbid psychiatric population's ascertainment. A study by Ko et al. 2014 reported variability in the proportion of symptoms and discussed the validity or diagnostic accuracy of IGD criteria. They reported that criterion 6 "continued excessive use of Internet gaming despite knowledge of psychosocial problems" and criterion 9 "jeopardized or lost a significant relationship, job, or educational or career opportunity" had the best diagnostic accuracy in differentiating the IGD group from the control group, followed by unsuccessful control, Tolerance, Preoccupation, withdrawal, escape, and loss of interest. Criterion 7 "deceiving" was the only criterion with a diagnostic accuracy of less than $75 \%$ (Ko et al., 2014). The role of various criteria in IGD should be further investigated in clinically diagnosed larger samples of IGD to ascertain to what extent this criterion is a useful indicator of disordered gaming or if these criteria may be best operationalized as a motivational factor for playing videogames? There may be a few potential explanations for these varied findings that merit consideration. On the one hand, these studies relied on different assessment tools to evaluate IGD. On the other hand, the samples recruited for these studies differed systematically in their basic demographic features and the different sampling techniques utilized across these studies.

The present study shows that the mean IGDS score as a measure of symptom severity was highest for Conflict (9.83 \pm 2.33$)$ followed by Persistence $(9.78 \pm 1.97)$, Withdrawal $(8.25 \pm 1.78)$, Tolerance (7.88 $\pm 1.58 \%)$, Preoccupation (7.81 $\pm 2.48 \%)$, problem (7.77 $\pm 1.78 \%)$, Deception (7.24 \pm 2.04$)$, Escape $(6.95 \pm 1.55 \%)$ and the least for Displacement (6.88 $1.55 \%)$. The study conducted by Király et al. (2017) concluded that "continuation" (Problem), "preoccupation", "negative consequences" (Conflict), and "escape" were more associated with lower levels of IGD while "tolerance", "loss of control" (Persistence), "give up other activities" (Displacement), and "deception" where more informative at higher levels of IGD 
(Király et al., 2017). The present study shows a pattern towards higher severity of IGD in the study sample when we compare the average scores of different criteria's of IGDS to see the severity of symptoms.

Association of IGD with psychiatric disorders has been well established in the literature(Andreassen et al., 2016; Bioulac et al., 2008; Chan \& Rabinowitz, 2006; Ferguson et al., 2011; D. Gentile, 2009; D. A. Gentile et al., 2011; Ko et al., 2006; Mazurek \& Engelhardt, 2013; Walther et al., 2012). Depression seems to be the most common comorbidity in all age groups(D. A. Gentile et al., 2011). In the present study, ODD (46.66\%) was most common psychiatric disorder associated with IGD followed by Dissociative disorder (24.44\%), ADHD (17.77\%) and Depressive disorder (11.11\%). In the present study, subjects diagnosed with Dissociative disorder having significantly higher IGDS scores for "Escape" criteria as compared to those with ADHD and Depression each. Online gamers get a more pleasant experience than offline gamers and, this might lead to a gradual and unconscious disconnection from reality. The phenomenon eventually leads to more brittle control over the individual's life and her self-awareness. The correlation between Internet gaming disorder risk and some dissociative experiences that emerge from a study by Concetta De Pasquale et al. (2018) shows a positive correlation between Internet gaming disorder risk and some dissociative experiences like depersonalization, derealization, absorption and imaginative involvement, and passive influence(De Pasquale et al., 2018). According to this study these people are presenting with disorders of affective and emotional regulation and may be more exposed to the risk of Internet gaming disorder, similarly as they are having a higher risk of other addictive disorders. These subjects are more prone to crave for "increasing levels of pleasure and involvement experienced within the gaming environment". Subjects having dissociative disorder engaged with gaming activity to escape the difficulty from psychosocial factors decreased social interaction(De Pasquale et al., 2018), frustration (Li et al., 2011), and life dissatisfaction (Li et al., 2011). Association of Female gender and Dissociative disorder may also explain the higher scores for "escape" in the present study. It has been well documented that there are gender differences found in the use of games and pathological gaming (Chiu et al., 2004; D. Gentile, 2009; Lee et al., 2009). In the present study, significantly higher IGDS scores found in females as compared to males in the "Escape" and the "Deception" criteria.

In present study Subjects with ODD were having significantly higher IGDS scores for "Displacement" criteria as compared to subjects diagnosed with Dissociative disorder. The literature shows that adolescents with high scores in IGD also have negative consequences at the psychosocial level: fewer recreational activities, fewer social activities and contacts, and diminished academic performance (Batthyany et al., 2009; Beutel et al., 2011). Generally, each online video game has an associated players' community which may lead players to find people online with similar interests and, thus, replace their "real-life" social network. As these online relationships spend more and more time, "real-world" social relations will tend to deteriorate or disappear, and this lack of "real-life" social support can lead some players to develop IGD. Subjects diagnosed with ODD plays more commonly violent video game. The highest proportion of ODD subjects can explain most of the present study subjects playing MMORPGs with violent or aggressive contents. Such kind of games gives more satisfaction and pleasure to these subjects. Excess involvement with such games may "displace" the previous hobbies and activities in these subjects. In the present study, the "Displacement" criterion was also significantly higher for urban 
subjects and subjects involved with online gaming. Online video games are never-ending, have global reach (Freeman, 2008; Daria J. Kuss \& Griffiths, 2012), attract gamers to play incessantly, and are associated with a greater tendency to develop addictive behaviours in contrast to offline video games (Internet Gaming Disorder, n.d.; Lemmens \& Hendriks, 2016). Due to faster internet facilities in urban regions, online gaming can also be considered another contributing factor to the "displacement"( $\mathrm{Li}$ et al., 2011). In the present study, the "Persistence" criterion was significantly higher for video games on personal devices and with the subjects involved with MMORPGs. The individual having personal devices can play video games anytime and anywhere without any interruption and permission. When attempts are made to stop this excessive gaming, they had withdrawal symptoms like irritability and aggression(Freeman, 2008). MMORPGs are the most preferred type of internet games, in which players often socialize in groups and co-operate to achieve game-relevant goals (Chiu et al., 2004).

The present study has certain limitations. This study shows that adolescents can be diagnosed with IGD based on DSM-5, but studies having control group comparison are needed to determine its applicability in Indian population. The study sample is clinic-based; patients were visiting OPD primarily for psychiatric disorders other than IGD. Although a correlation has been found between psychiatric disorders and IGD symptoms, yet, a cause-effect relationship cannot be established with this study design. The strengths include that both the parents and adolescents have been interviewed while the previous studies have used either adolescents or parents' responses by self-rated forms or checklists (King \& Delfabbro, 2016; Király et al., 2017; Pontes \& Griffiths, 2015). In this study, the phenomenology of DSM-5 internet gaming disorders (IGD-DSM-5) and associated psychiatric disorders have been discussed in a small clinical population that needs to be examined by large sample prospective, community-based multi-cantered clinical studies.

The present study is the first Indian study that explored the phenomenology of IGD in terms of using DSM-5 IGD criteria, symptom severity, and its association with psychosocial variables, specifically the variables of game playing behaviours like the accessibility of device, mode of the game, and type of games. This study suggests that symptoms of IGD have a significant association with factors like gender, domicile, gaming genre (MMORPGs), accessibility of smartphones, online/offline modes of gaming and the associated psychiatric comorbidity. These factors can be viewed in terms of modifiable risk factors and have treatment implications.

\section{Abbreviations}

IGD- Internet Gaming Disorder

DSM-5- Diagnostic and Statistical Manual of Mental disorder

IGDS- Internet Gaming Disorder Scale

K-SADS-PL- Kiddie Schedule for Affective Disorders and Schizophrenia for School-Age Children-Present and Lifetime version 
ADHD- Attention Deficit Hyperactivity Disorder

MMORPGs- Massive Multiplayer Online Role-Playing Games

\section{Declarations}

\section{Disclosures and declerartions:}

Authors PS, AA, SS, PKG, VA, have no potential conflicts of interests to be disclosed

'All procedures followed were in accordance with the ethical standards of the responsible committee on human experimentation (institutional and national) and with the Helsinki Declaration of 1975, as revised in 2000 (5). Informed consent was obtained from all patients for being included in the study.'

Disclosure for funding: Authors Dr. Praveen Sachan, Dr. Amit Arya, Shweta Singh, Pawan Kumar Gupta, Dr. Vivek Agarwal did not receive support from any organization for the submitted work. No funding was received to assist with the preparation of this manuscript. No funding was received for conducting this study. No funds, grants, or other support was received. The authors have no relevant financial or nonfinancial interests to disclose.

Conflict of interest: The authors have no conflicts of interest to declare that are relevant to the content of this article. All authors certify that they have no affiliations with or involvement in any organization or entity with any financial interest or non-financial interest in the subject matter or materials discussed in this manuscript. The authors have no financial or proprietary interests in any material discussed in this article.

Declaration for Informed consent: 'All procedures followed were in accordance with the ethical standards of the responsible committee on human experimentation (institutional and national) and with the Helsinki Declaration of 1975, as revised in 2000 (5). Informed consent was obtained from all patients for being included in the study.'

Ethical Approval: The study is approved by the Institutional Ethics Committee of King George's Medical University, Lucknow (Ref. Code:97 ${ }^{\text {Th }}$ ECM II B-Thesis/P141).

The authors of this article had access to all study data, are responsible for all contents of the article, had authority over manuscript preparation, and decided to submit the manuscript for publication. All listed authors have approved of the submission of the manuscript to the journal.

\section{References}

Andreassen, C. S., Billieux, J., Griffiths, M. D., Kuss, D. J., Demetrovics, Z., Mazzoni, E., \& Pallesen, S. (2016). The relationship between addictive use of social media and video games and symptoms of psychiatric disorders: A large-scale cross-sectional study. Psychology of Addictive Behaviors, 30(2), 252. 
Association, A. P. (2013). Diagnostic and statistical manual of mental disorders (DSM-5®). American Psychiatric Pub.

Batthyany, D., Müller, K. W., Benker, F., \& Woelfling, K. (2009). Computer game playing: Clinical characteristics of dependence and abuse among adolescents. Wiener Klinische Wochenschrift, 121(15/16), 502-509.

Beutel, M. E., Hoch, C., Wölfling, K., \& Müller, K. W. (2011). Clinical characteristics of computer game and internet addiction in persons seeking treatment in an outpatient clinic for computer game addiction. Zeitschrift Fur Psychosomatische Medizin Und Psychotherapie, 57(1), 77-90.

Bioulac, S., Arfi, L., \& Bouvard, M. P. (2008). Attention deficit/hyperactivity disorder and video games: A comparative study of hyperactive and control children. European Psychiatry, 23(2), 134-141.

Chan, P. A., \& Rabinowitz, T. (2006). A cross-sectional analysis of video games and attention deficit hyperactivity disorder symptoms in adolescents. Annals of General Psychiatry, 5(1), 16.

Chiu, S.-I., Lee, J.-Z., \& Huang, D.-H. (2004). Video game addiction in children and teenagers in Taiwan. CyberPsychology \& Behavior, 7(5), 571-581.

De Pasquale, C., Dinaro, C., \& Sciacca, F. (2018). Relationship of Internet gaming disorder with dissociative experience in Italian university students. Annals of General Psychiatry, 17(1), 28.

Domino, G., \& Domino, M. L. (2006). Psychological testing: An introduction. Cambridge University Press.

Dong, G., \& Potenza, M. N. (2014). A cognitive-behavioral model of Internet gaming disorder: Theoretical underpinnings and clinical implications. Journal of Psychiatric Research, 58, 7-11.

Ferguson, C. J., Coulson, M., \& Barnett, J. (2011). A meta-analysis of pathological gaming prevalence and comorbidity with mental health, academic and social problems. Journal of Psychiatric Research, 45(12), 1573-1578.

Freeman, C. B. (2008). Internet gaming addiction. The Journal for Nurse Practitioners, 4(1), 42-47.

Gentile, D. (2009). Pathological video-game use among youth ages 8 to 18: A national study. Psychological Science, 20(5), 594-602.

Gentile, D. A., Choo, H., Liau, A., Sim, T., Li, D., Fung, D., \& Khoo, A. (2011). Pathological video game use among youths: A two-year longitudinal study. Pediatrics, 127(2), e319-e329.

Griffiths, M. D., Kuss, D. J., \& King, D. (2013). Video game addiction: Past, present and future. Extended session: Playing with fire? Intense game experiences and discussions and debates in pathological gaming. 
Griffiths, M., \& Wood, R. T. (2000). Risk factors in adolescence: The case of gambling, videogame playing, and the Internet. Journal of Gambling Studies, 16(2-3), 199-225.

Internet Gaming Disorder: Investigating a proposed non-substance related addiction among medical undergraduates-a cross-sectional study. (n.d.). Retrieved January 11, 2021, from

https://1 library.net/document/zx92kvnz-internet-disorder-investigating-proposed-substance-addictionundergraduates-sectional.html

Jiang, Q., \& Leung, L. (2012). Effects of individual differences, awareness-knowledge, and acceptance of Internet addiction as a health risk on willingness to change Internet habits. Social Science Computer Review, 30(2), 170-183.

Kaufman, J., Birmaher, B., Brent, D., Rao, U. M. A., Flynn, C., Moreci, P., Williamson, D., \& Ryan, N. (1997). Schedule for affective disorders and schizophrenia for school-age children-present and lifetime version (K-SADS-PL): Initial reliability and validity data. Journal of the American Academy of Child \& Adolescent Psychiatry, 36(7), 980-988.

King, D. L., \& Delfabbro, P. H. (2016). The cognitive psychopathology of Internet gaming disorder in adolescence. Journal of Abnormal Child Psychology, 44(8), 1635-1645.

Király, O., Sleczka, P., Pontes, H. M., Urbán, R., Griffiths, M. D., \& Demetrovics, Z. (2017). Validation of the ten-item Internet Gaming Disorder Test (IGDT-10) and evaluation of the nine DSM-5 Internet Gaming Disorder criteria. Addictive Behaviors, 64, 253-260.

Király, O., Urbán, R., Griffiths, M. D., Ágoston, C., Nagygyörgy, K., Kökönyei, G., \& Demetrovics, Z. (2015). The mediating effect of gaming motivation between psychiatric symptoms and problematic online gaming: An online survey. Journal of Medical Internet Research, 17(4), e88.

Ko, C.-H., Yen, J.-Y., Chen, C.-C., Chen, S.-H., Wu, K., \& Yen, C.-F. (2006). Tridimensional personality of adolescents with internet addiction and substance use experience. The Canadian Journal of Psychiatry, 51(14), 887-894.

Ko, C.-H., Yen, J.-Y., Chen, S.-H., Wang, P.-W., Chen, C.-S., \& Yen, C.-F. (2014). Evaluation of the diagnostic criteria of Internet gaming disorder in the DSM-5 among young adults in Taiwan. Journal of Psychiatric Research, 53, 103-110.

Kuss, Daria J., \& Griffiths, M. D. (2012). Online gaming addiction in children and adolescents: A review of empirical research. Journal of Behavioral Addictions, 1(1), 3-22.

Kuss, Daria Joanna, \& Griffiths, M. D. (2012). Internet gaming addiction: A systematic review of empirical research. International Journal of Mental Health and Addiction, 10(2), 278-296.

Lee, S.-J., Bartolic, S., \& Vandewater, E. A. (2009). Predicting children's media use in the USA: Differences in cross-sectional and longitudinal analysis. British Journal of Developmental Psychology, 27(1), 123- 
Leeman, R. F., \& Potenza, M. N. (2013). A targeted review of the neurobiology and genetics of behavioural addictions: An emerging area of research. The Canadian Journal of Psychiatry, 58(5), 260-273.

Lemmens, J. S., \& Hendriks, S. J. (2016). Addictive online games: Examining the relationship between game genres and Internet Gaming Disorder. Cyberpsychology, Behavior, and Social Networking, 19(4), 270-276.

Lemmens, J. S., Valkenburg, P. M., \& Gentile, D. A. (2015). The Internet gaming disorder scale. Psychological Assessment, 27(2), 567.

Li, D., Liau, A., \& Khoo, A. (2011). Examining the influence of actual-ideal self-discrepancies, depression, and escapism, on pathological gaming among massively multiplayer online adolescent gamers. Cyberpsychology, Behavior, and Social Networking, 14(9), 535-539.

Mazurek, M. O., \& Engelhardt, C. R. (2013). Video game use in boys with autism spectrum disorder, ADHD, or typical development. Pediatrics, 132(2), 260-266.

Mihara, S., \& Higuchi, S. (2017). Cross-sectional and longitudinal epidemiological studies of I nternet gaming disorder: A systematic review of the literature. Psychiatry and Clinical Neurosciences, 71(7), 425444.

Pontes, H. M., \& Griffiths, M. D. (2015). Measuring DSM-5 Internet gaming disorder: Development and validation of a short psychometric scale. Computers in Human Behavior, 45, 137-143.

Rehbein, F., Zenses, E.-M., Möller, C., \& Mößle, T. (2015). Computerspielabhängigkeit im Jugendalter. Monatsschrift Kinderheilkunde, 163(7), 701-705.

Rehbein, Florian, \& Baier, D. (2013). Family-, media-, and school-related risk factors of video game addiction. Journal of Media Psychology.

Rehbein, Florian, Kliem, S., Baier, D., Mößle, T., \& Petry, N. M. (2015). Prevalence of internet gaming disorder in German adolescents: Diagnostic contribution of the nine DSM-5 criteria in a state-wide representative sample. Addiction, 110(5), 842-851.

Walther, B., Morgenstern, M., \& Hanewinkel, R. (2012). Co-occurrence of addictive behaviours: Personality factors related to substance use, gambling and computer gaming. European Addiction Research, 18(4), 167-174.

Weinstein, A., \& Lejoyeux, M. (2015). New developments on the neurobiological and pharmaco-genetic mechanisms underlying internet and videogame addiction. The American Journal on Addictions, 24(2), 117-125. 